\title{
Preventing glycaemic relapse in recently controlled type 2 diabetes patients: a randomised controlled trial
}

\author{
M. M. Huizinga • T. Gebretsadik • C. Garcia Ulen • \\ A. K. Shintani • S. R. Michon • L. O. Shackleford • \\ K. L. Wolff • A. W. Brown • R. L. Rothman • T. A. Elasy
}

Received: 24 August 2009 / Accepted: 16 December 2009 / Published online: 19 January 2010

(C) Springer-Verlag 2010

\begin{abstract}
Aims/hypothesis After achieving glycaemic control, many type 2 diabetic patients relapse to clinically significant levels of hyperglycaemia. We sought to determine the optimal frequency of telephone contact by nurse practitioners that was necessary to prevent glycaemic relapse.
\end{abstract}

Electronic supplementary material The online version of this article (doi:10.1007/s00125-010-1658-3) contains supplementary material, which is available to authorised users.

A. K. Shintani · S. R. Michon · L. O. Shackleford · K. L. Wolff ·

A. W. Brown $\cdot$ R. L. Rothman $\cdot$ T. A. Elasy

Diabetes Research and Training Center,

Vanderbilt University Medical Center,

Nashville, TN, USA

S. R. Michon • L. O. Shackleford • R. L. Rothman • T. A. Elasy Division of General Internal Medicine and Public Health, Department of Medicine, Center for Health Services Research, Vanderbilt University Medical Center,

Nashville, TN, USA

M. M. Huizinga $(\square)$

Johns Hopkins University School of Medicine,

2024 E Monument Street, Suite 2-500,

Baltimore, MD 21205, USA

e-mail: mhuizinga@jhmi.edu

T. A. Elasy

VA Tennessee Valley Healthcare System,

Nashville, TN, USA

T. Gebretsadik $\cdot$ A. K. Shintani

Department of Biostatistics,

Vanderbilt University Medical Center,

Nashville, TN, USA

C. Garcia Ulen

University of Michigan School of Medicine,

Ann Arbor, MI, USA
Methods This parallel, randomised controlled trial ran from June 2002 to February 2006 at an academic medical centre, studying 164 type 2 diabetic patients who had recently achieved glycaemic control. Participants were randomly assigned by sequential, concealed, computer-generated allocation to a 2 year maintenance strategy consisting of: (1) routine follow-up $(n=54)$; (2) routine follow-up and quarterly telephone contact $(n=55)$; or (3) routine followup and monthly telephone contact $(n=55)$. Blinding was not possible. The primary outcome was cumulative incidence of glycaemic relapse, defined as an increase in $\mathrm{HbA}_{1 \mathrm{c}}$ of $\geq 1 \%$; all participants were analysed. Cumulative incidence and prevalent proportions were compared. Weight change and hypoglycaemia were also assessed.

Results All participants randomised were included in the analyses. The study was completed by $90 \%$ of participants and intervention fidelity was high. At 24 months, the cumulative incidence of relapse was $41 \%$. At 12 months, prevalent proportions of relapse were $20 \%, 14 \%$ and $15 \%$ for control, quarterly contact and monthly contact, respectively. At 24 months, they were $25 \%, 21 \%$ and $29 \%$, respectively. There was no statistically significant difference in cumulative incidence or prevalent proportions of relapse among the study arms. Adverse events did not differ between study arms.

Conclusions/interpretation This first randomised controlled trial to test an intervention to prevent glycaemic relapse found that regularly scheduled telephone contact by a nurse practitioner was no more effective than routine follow-up care in preventing glycaemic relapse.

Trial registration: ClinicalTrials.gov NCT00362193

Funding: The research was supported by the National Institute of Diabetes and Digestive and Kidney Disease R18 DK 062258, P60 DK 020593 and K24 DK 077875. M. M. 
Huizinga was supported by National Institute of Environmental Health Sciences 1 K12 ES 015855 and National Center for Research Resources 5 K12 RR 023266.

Keywords Behavioural intervention · Diabetes ·

Haemoglobin $\mathrm{A}_{1 \mathrm{c}} \cdot$ Long-term care .

Randomised controlled trial $\cdot$ Relapse

\section{Abbreviations \\ DIP Diabetes improvement programme \\ GEE Generalised estimating equations \\ IQR Interquartile range}

\section{Introduction}

Large randomised controlled trials have demonstrated that glycaemic control reduces the incidence of long-term complications of diabetes [1]. Yet $43 \%$ of patients with diabetes are unable to meet the recommendation of the American Diabetes Association to achieve $\mathrm{HbA}_{1 \mathrm{c}}$ of less than 7\% [2].

Suboptimal management of diabetes has prompted calls from the Institute of Medicine and Congress for quality improvement and the development of alternative models of healthcare delivery [3]. A new industry, disease management, has emerged to fill these quality gaps. There is scant evidence that these efforts translate into long-term improvements in glycaemic control [4]. Clinic-based interventions focusing on medication intensification, provider education and patient reminders by nurses or other mid-level providers are effective for achieving glycaemic control in patients with diabetes [5]. However, patients often relapse to clinically significant levels of hyperglycaemia within 1 to 2 years [6-8]. Although prevention of relapse is important in chronic disease management, glycaemic relapse prevention has received little attention or critical analysis, either in the literature or from the disease management industry [9].

The primary aim of this study was to assess the relative effectiveness of a case-management follow-up strategy for preventing glycaemic relapse after glycaemic control had been achieved. The study sought to determine the optimal frequency of telephone contact by nurse practitioners that would be necessary to prevent glycaemic relapse in patients with type 2 diabetes. It was hypothesised that glycaemic relapse would be prevented in a dose-dependent fashion, with higher relapse prevention in the arm that had the most frequent patient contacts.

\section{Methods}

Design We conducted a randomised controlled trial to assess the efficacy of a 2 year programme to prevent glycaemic relapse in patients with recently controlled type 2 diabetes. The study protocol has been previously described [10]. Following completion of a 3 month intensive diabetes improvement programme (DIP), participants with a $\geq 1 \%$ decrease in $\mathrm{HbA}_{1 \mathrm{c}}$ were randomised to the following maintenance regimens: (1) routine follow-up in a primary care clinic (control); (2) routine follow-up with quarterly telephone contact; or (3) routine follow-up with monthly telephone contact.

Setting Participants were recruited from an urban area surrounding an academic medical centre in Nashville, Tennessee. Participants were enrolled from June 2002 to January 2005. Telephone contacts were handled by an academic centre clinic.

Participants Patients who had been referred to the diabetes DIP by their primary care physician or endocrinologist for poor glycaemic control $\left(\mathrm{HbA}_{1 \mathrm{c}}>8 \%\right)$ and who demonstrated significant improvement $\left(\geq 1 \%\right.$ decline in $\left.\mathrm{HbA}_{1 \mathrm{c}}\right)$ during the DIP were recruited for the study. The DIP is a usual-care diabetes management programme offered within the academic medical centre and consists of a physician-led team, including a nurse practitioner, a registered dietitian and a diabetes nurse, and has been described previously [11-13]. The goal of the DIP is to achieve glycaemic control through medication titration and adherence, physical activity, medical nutrition therapy and self-monitoring of blood glucose. Eligibility criteria included age 18 to 75 years and being English-speaking. Pregnant women were excluded.

Randomisation and intervention Within 2 weeks of completing the DIP, patients were contacted. If patients met inclusion criteria and provided informed consent, eligibility was confirmed and the patient was invited to participate. Randomisation was performed using a computerised randomisation process and assignments, which were concealed, were obtained sequentially from a computer program, as has been previously described [10]. Blinding to assignment was not possible given the nature of the study.

The intervention consisted of a phone contact by a nurse practitioner with referral to a dietitian if nutrition self-care was perturbed. The nurse practitioners and dietitians were all certified in diabetes education (Certified Diabetes Educators [CDE] or Advance Diabetes Management-Board Certified [ADM-BC]) and had significant clinical experience in providing diabetes care. For the study, the nurse practitioners adhered to a set of intervention protocols and guidelines, which have been described in detail in a separate manuscript [10]. Providers helped participants titrate medications, and identify and solve problems arising in self-care behaviours, including diet, exercise, self-monitoring of blood glucose and medication adherence. If no problems in self-care 
behaviour were identified, anticipatory planning, positive reinforcement and mutually established goal-setting were performed. If a problem in a self-care behaviour was identified and the participant was able to identify the source of the problem, then a standard problem solving paradigm was employed. If the source of the problem could not be identified, then the provider either engaged in motivational interviewing to further explore the problem or compensated for failure to identify the problem by altering another selfcare behaviour as a temporary measure until the source of the original problem could be identified. Only the frequency of the intervention varied between treatment arms.

To enhance the reliability and validity of the intervention, intervention fidelity tools were used to monitor the phone contacts between the nurse practitioners and study participants [14]. The analysis consisted of standard, qualitative descriptions of the extent to which the intervention phone calls were consistent with defined intervention protocol and guidelines. Consistency between the nurse practitioners was also determined. We randomly selected $10 \%$ of the phone calls and audio-taped them. Raters used checklists derived from protocols to document which elements were conducted or omitted. Intervention fidelity was high (all elements of the protocol present in $>80 \%$ of the calls assessed) and did not differ between nurse practitioners [10].

Participants in the intervention arms were asked to rate their satisfaction with the intervention and the nurse practitioner on a five point Likert scale. They were also asked to give qualitative feedback about the intervention.

Outcomes and measurements The primary outcome was glycaemic relapse defined as an increase in $\mathrm{HbA}_{1 \mathrm{c}}$ of $\geq 1 \%$ over baseline. $\mathrm{HbA}_{1 \mathrm{c}}$ was assessed at baseline and at each follow-up visit $\left(3,6,12,18\right.$ and 24 months). $\mathrm{HbA}_{1 \mathrm{c}}$ measurement was conducted at Vanderbilt University, which has received a certificate of traceability to the DCCT reference method by the National Glycohemoglobin Standardization Program to ensure precision and accuracy of this measurement. Secondary outcomes were also assessed but are not described in this manuscript [10].

Adverse outcomes were also assessed at each follow-up visit. Frequency and severity of hypoglycaemia were assessed by the survey questions: 'In the last month, how many times have you had symptoms of low blood sugar?' and 'In the last month, how many times has someone had to help you because of low blood sugar?' Severe hypoglycaemia was defined as hypoglycaemia requiring assistance. Weight change was measured as per cent change from baseline weight. Participants were weighed in street clothes, without shoes or heavy overgarments, using a platform scale (Detecto balance scale, Webb City, MO, USA) and recorded to the nearest tenth of a pound. Weight was converted to $\mathrm{kg}$ for analyses.
Statistical analysis Sample size calculation was performed based on $\chi^{2}$ test for linear trend in proportions of patients among the three study arms who were expected to relapse during the study period. Based on previous observational work, we expected patients to relapse as follows during the study period: $50 \%$ of the control arm (routine primary care follow-up); $30 \%$ of the quarterly contact arm; and $20 \%$ of the monthly contact arm $[7,8]$. Anticipating $20 \%$ attrition, 165 participants were expected to provide $85 \%$ power to detect a statistically significant linear trend at two-sided $5 \%$ alpha level. Calculations for power analysis were performed using nQuery Advisor version 4.0 (Statistical Solutions, Saugus, MA, USA). One participant was inappropriately randomised and not included in the analyses. This participant was found to have type 1 diabetes after randomisation and was excluded on the basis of study exclusion criteria.

The primary outcome was analysed in two ways. Cumulative incidence proportion of glycaemic relapse over 24 months was estimated using Kaplan-Meier analysis and compared with the logrank test between treatment groups. The prevalent proportions of relapse over 24 months for each study group were compared by generalised estimating equations (GEE) methods.

The secondary outcome of weight change from baseline to 24 months was compared between the study arms using linear mixed effects models. Prevalence proportions of hypoglycaemia and severe hypoglycaemia were assessed by GEE methods by status over 24 months. To identify the effect of the intervention in specific subgroups of patients, exploratory analyses of the primary outcome were performed for post hoc subgroups of age, sex, race, baseline BMI, baseline insulin use and baseline duration of diabetes. Odds ratio estimates and $95 \%$ CI were generated for the subgroups of interest by GEE methods while adjusting for age, baseline BMI (dichotomised as $<35$ or $\geq 35 \mathrm{~kg} / \mathrm{m}^{2}$ ) and sex. $p$ values for interaction were assessed between status and all the covariates included in the model and were not statistically significant. All statistical analyses were performed using R version 2.6.3 (R Foundation for Statistical Computing, Vienna, Austria).

Ethics All participants provided written, informed consent. The study protocol was approved by the Institutional Review Board at Vanderbilt University. The funding agency had no role in the design, conduct, analysis or decision to publish.

\section{Results}

We enrolled 164 patients in the study (Fig. 1). Of these, $44 \%$ were women, $21 \%$ were African-American and $89 \%$ had completed at least a high school degree. At baseline, 


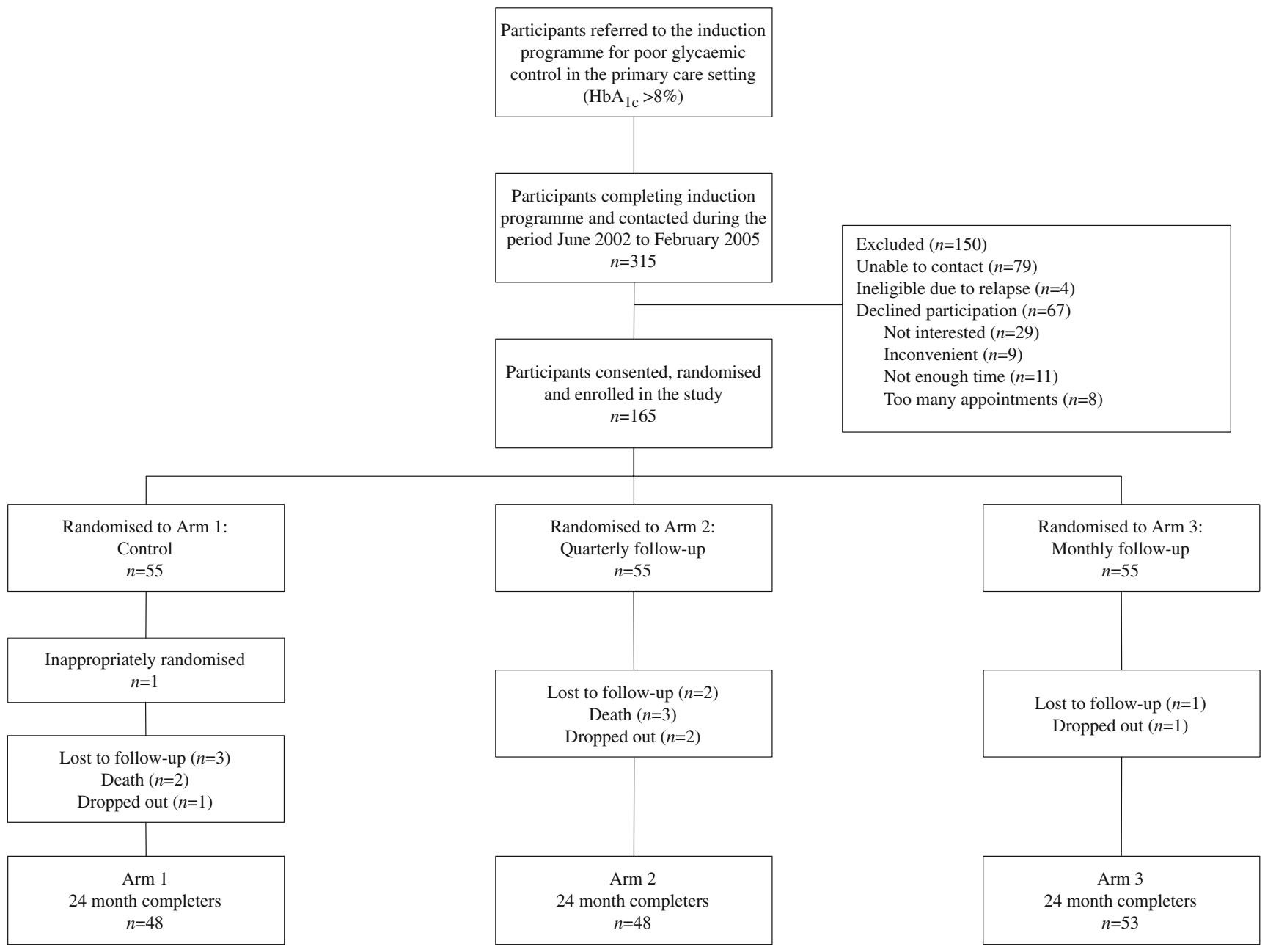

Fig. 1 Participant flow throughout the study. Lost to follow-up denotes an inability of the study staff to contact the participant; dropped out refers to those participants who asked to no longer participate in the study

the mean (SD) age was 55 (11) years, $\mathrm{HbA}_{1 \mathrm{c}}(\mathrm{SD})$ was $6.7 \%(0.7)$ and BMI (SD) was $34(7) \mathrm{kg} / \mathrm{m}^{2}$. At baseline $54 \%$ of participants reported insulin use. The median (interquartile range [IQR]) duration of diabetes was 5 $(0.5-10)$ years. Differences in baseline demographic and disease-based characteristics were not statistically significant across treatment groups (Table 1).

Intervention implementation The median follow-up time was 24 months, with $90 \%$ completing the 2-year follow-up; there was no difference between the study arms with regard to follow-up completion. Overall, adherence to the protocol was quite high with all elements present in $79 \%$ of sampled interviews. The educators did not differ significantly in any category. In the intervention arms, approximately $93 \%$ of the scheduled telephone contacts were completed. Participants reported high satisfaction with the intervention.

Glycaemic relapse The prevalence of relapse at 3 months was $6 \%, 9 \%$ and $8 \%$ for the control, quarterly contact and monthly contact arms, respectively (Table 2). At 24 months, the prevalence of relapse was $25 \%, 21 \%$ and $29 \%$ for the three arms, respectively. Relapse prevalence proportions did not differ between treatment groups over follow-up time ( $p=0.83$ for status and time interaction). At 24 months, the cumulative incidence of relapse was $41 \%$. The cumulative incidence proportions did not differ between the treatment groups (logrank, $p=0.72$; Fig. 2).

Adverse outcomes The incidence of hypoglycaemia and severe hypoglycaemia did not differ significantly across treatment groups over follow-up visits time point $(p=0.57$ for hypoglycaemia; $p=0.56$ for severe hypoglycaemia; Electronic supplementary material [ESM] Table 1). Throughout the study, $50 \%$ to $64 \%$ of participants reported a hypoglycaemic event during the previous month. Only $4 \%$ to $8 \%$ reported experiencing a severe hypoglycaemic event in the previous month. At study completion, mean weight change (SD) was 1.37 (7.28) $\mathrm{kg}$. Weight change did not differ between study arms ( $p=0.54$, Kruskal-Wallis). 
Table 1 Baseline characteristics of the study population

\begin{tabular}{|c|c|c|c|c|c|}
\hline Characteristic & All & Control & Quarterly contact & Monthly contact & $p$ value $\mathrm{e}^{\mathrm{a}, \mathrm{b}}$ \\
\hline$n$ & 164 & 54 & 55 & 55 & \\
\hline Age (years), mean (SD) & $55.1(10.7)$ & $56.2(9.7)$ & $55.7(11.4)$ & $53.5(11)$ & 0.47 \\
\hline Women, $n(\%)$ & $72(44)$ & $23(43)$ & $21(38)$ & $28(51)$ & 0.39 \\
\hline African-American, $n(\%)$ & $35(21)$ & $7(13)$ & $16(29)$ & $12(22)$ & 0.12 \\
\hline Education $\geq \mathrm{HS}, n(\%)$ & $146(89)$ & 47 (87) & $49(89)$ & $50(91)$ & 0.81 \\
\hline $\mathrm{HbA}_{1 \mathrm{c}}(\%)$, mean $(\mathrm{SD})$ & $6.7(0.68)$ & $6.65(0.71)$ & $6.63(0.74)$ & $6.78(0.6)$ & 0.47 \\
\hline Diabetes duration (years), median (IQR) & $5(0.5-10)$ & $5.5(0.67-10)$ & $4(0.55-9.5)$ & $4(0.5-10)$ & 0.78 \\
\hline BMI $\left(\mathrm{kg} / \mathrm{m}^{2}\right)$, mean (SD) & $34(6.9)$ & $34.1(7.2)$ & $33.1(6.2)$ & $34.9(7.3)$ & 0.66 \\
\hline Waist circumference $(\mathrm{cm})$, mean (SD) & $109(15)$ & $111(16)$ & $106(12)$ & $110(16)$ & 0.39 \\
\hline Insulin use, $n(\%)$ & $89(54)$ & $32(59)$ & $25(45)$ & $32(58)$ & 0.27 \\
\hline Units/kg, median (IQR) & $55(25-92)$ & $39(24-79)$ & $59(32-100)$ & $61(25-93)$ & 0.78 \\
\hline Shots/day, median (IQR) & $3(1-4)$ & $2.5(1-4)$ & $2(2-4)$ & $3(1-4)$ & 0.56 \\
\hline Short-acting, $n(\%)$ & $30(34)$ & $8(24)$ & $10(31)$ & $12(52)$ & 0.04 \\
\hline Long-acting, $n(\%)$ & $39(44)$ & $19(56)$ & $8(25)$ & $12(52)$ & 0.11 \\
\hline Oral medication use, $n(\%)$ & $118(72)$ & $37(69)$ & $41(75)$ & $40(73)$ & 0.41 \\
\hline Metformin, $n(\%)$ & $98(60)$ & $32(59)$ & $32(58)$ & $34(62)$ & 0.94 \\
\hline Sulfonylurea, $n(\%)$ & $67(41)$ & $22(41)$ & $25(45)$ & $20(36)$ & 0.63 \\
\hline Thiazolidinedione, $n(\%)$ & $21(13)$ & $11(20)$ & $8(15)$ & $2(4)$ & 0.72 \\
\hline Meglitinides, $n(\%)$ & $5(3)$ & $2(4)$ & $1(2)$ & $2(4)$ & 0.61 \\
\hline Systolic BP (mmHg), mean (SD) & $126(16)$ & $126(15)$ & $125(17)$ & $127(15)$ & 0.64 \\
\hline Diastolic BP (mmHg), mean (SD) & $73(11)$ & $72(9)$ & $72(11)$ & $73(12)$ & 0.80 \\
\hline \multicolumn{6}{|l|}{ Lipids } \\
\hline LDL (mmol/l), mean (SD) & $2.5(0.8)$ & $2.5(0.7)$ & $2.5(0.8)$ & $2.5(0.8)$ & 0.94 \\
\hline HDL (mmol/l), mean (SD) & $1.1(0.3)$ & $1.1(0.3)$ & $1.1(0.3)$ & $1.1(0.3)$ & 0.57 \\
\hline Triacylglycerol (mmol/l), median (IQR) & $1.9(1.4-2.7)$ & $2.1(1.4-2.6)$ & $1.9(1.4-2.8)$ & $1.8(1.3-2.5)$ & 0.80 \\
\hline CESD score, median (IQR) & $9(4-17)$ & $9(4-18)$ & $10.5(4-17)$ & $7(4-17)$ & 0.83 \\
\hline
\end{tabular}

${ }^{\text {a }} F$ statistic from Kruskal-Wallis test used to identify statistically significant differences between groups for continuous variables

${ }^{\mathrm{b}}$ Pearson test used to identify statistically significant differences between groups for categorical variables

CESD, Center for Epidemiologic Studies Depression Scale; HS, high school education

Subgroup analyses Exploratory subgroup analyses are shown in Fig. 3. Participants with stage 2 or 3 obesity (BMI $\geq 35 \mathrm{~kg} /$ $\mathrm{m}^{2}$ ) were less likely to relapse in the intervention arms in a dose-dependent fashion (quarterly contact OR 0.5, 95\% CI 0.2-1.6; monthly contact: OR $0.4,95 \%$ CI $0.2-0.9$ ). However, participants with $\mathrm{BMI}<35 \mathrm{~kg} / \mathrm{m}^{2}$ tended to be more likely to relapse in the intervention arms than in the control arm; in them relapse was also dose-dependent with harm in- creasing with more frequent contact (quarterly contact OR 1.8, 95\% CI 0.7-4.6; monthly contact OR 2.4, 95\% CI 0.9-6.3).

\section{Discussion}

In a single centre randomised controlled trial, regularly scheduled telephone contact by a nurse practitioner was no
Table 2 Relapse prevalent proportion at each follow-up time point

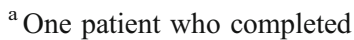
24 month follow-up did not have $\mathrm{HbA}_{1 \mathrm{c}}$ data for that time-point
}

\begin{tabular}{lllll}
\hline Variables & All & Control & Quarterly contact & Monthly contact \\
\hline $\begin{array}{l}\text { Group size }(n) \\
\text { Follow-up time period (months) }\end{array}$ & 164 & 54 & 55 & 55 \\
$3, \%(n / n)$ & $8(12 / 158)$ & $6(3 / 52)$ & $9(5 / 53)$ & $8(4 / 53)$ \\
$6, \%(n / n)$ & $14(21 / 151)$ & $12(6 / 48)$ & $14(7 / 51)$ & $15(8 / 52)$ \\
$12, \%(n / n)$ & $16(25 / 152)$ & $20(10 / 49)$ & $14(7 / 49)$ & $15(8 / 54)$ \\
$18, \%(n / n)$ & $21(30 / 142)$ & $20(9 / 45)$ & $20(9 / 46)$ & $24(12 / 51)$ \\
$24, \%(n / n)$ & $25(37 / 148)$ & $25(12 / 48)$ & $21(10 / 48)$ & $29(15 / 52)^{\mathrm{a}}$ \\
\hline
\end{tabular}




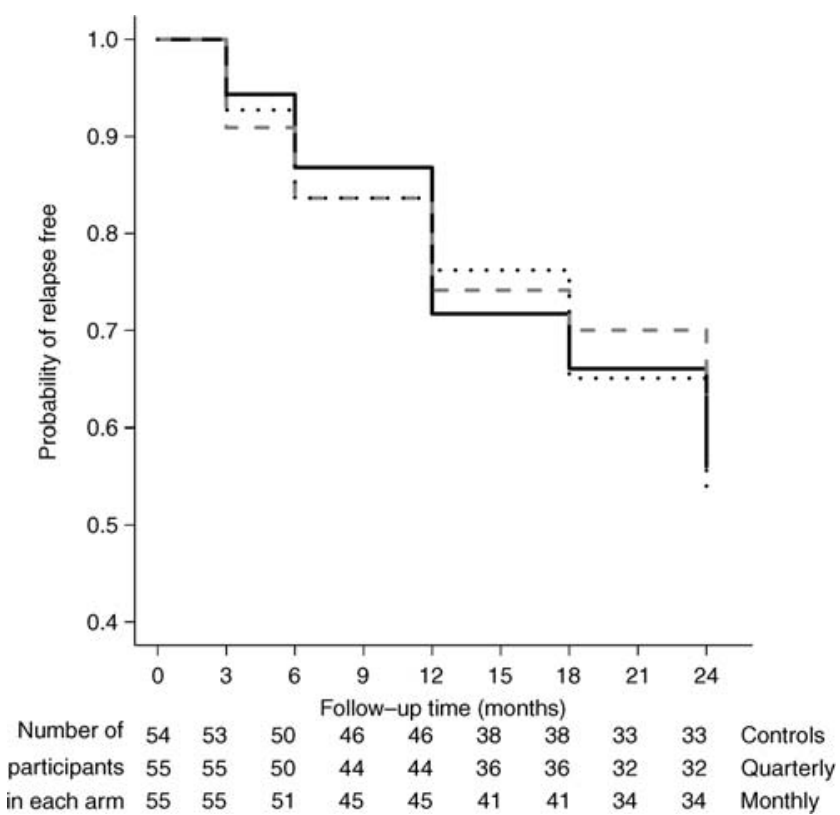

Fig. 2 Survival analysis of time of first relapse. No difference was detected between the study arms ( $p=0.72$, logrank). Continuous line, control; dotted line, monthly; dashed line, quarterly

more effective than routine follow-up care in preventing glycaemic relapse in type 2 diabetes patients who had recently achieved glycaemic control. Adverse events, including hypoglycaemia, severe hypoglycaemia and weight change also did not differ across treatment groups. The intervention aimed to provide reminders, titrate medications and solve problems arising in self-care behaviours. The intervention was delivered as planned and had high intervention fidelity. We had a very low attrition rate and, overall, participants were highly satisfied with the intervention.

This is the first study in the diabetes quality improvement literature that concentrates on prevention of glycaemic relapse. Prior efforts have focused on improving the quality of diabetes care for uncontrolled patients or primary care populations as a whole [5]. A recent review of diabetes quality improvement initiatives found that team changes, including expanding the role of nurse practitioners, and case management programmes are both able to improve glycaemic control, especially when case managers are able to adjust medications without awaiting physician approval [15]. However, the incremental impact of interventions was greatly reduced among patients with $\mathrm{HbA}_{1 \mathrm{c}}<8 \%$, suggesting that the approach or the content of care was not adequately targeted towards this population.

The present study incorporated team changes and a case management approach, but also included a patient education component. During regularly scheduled telephone contacts by nurse practitioners, participants who had selfidentified problems in self-care were led through problemsolving steps and coping strategies; if no problems were self-identified, anticipatory planning, goal setting and positive reinforcement were emphasised. Nurse practitioners also adjusted medications as necessary. However, no specific training in problem-solving or relapse prevention techniques was provided to the patients during the DIP programme that preceded the randomisation. This lack of preparation during the improvement phase of care may
Fig. 3 Post hoc subgroup analysis of glycaemic relapse. Circles, quarterly contact; diamonds, monthly contact. ${ }^{\mathrm{a}}$ At baseline

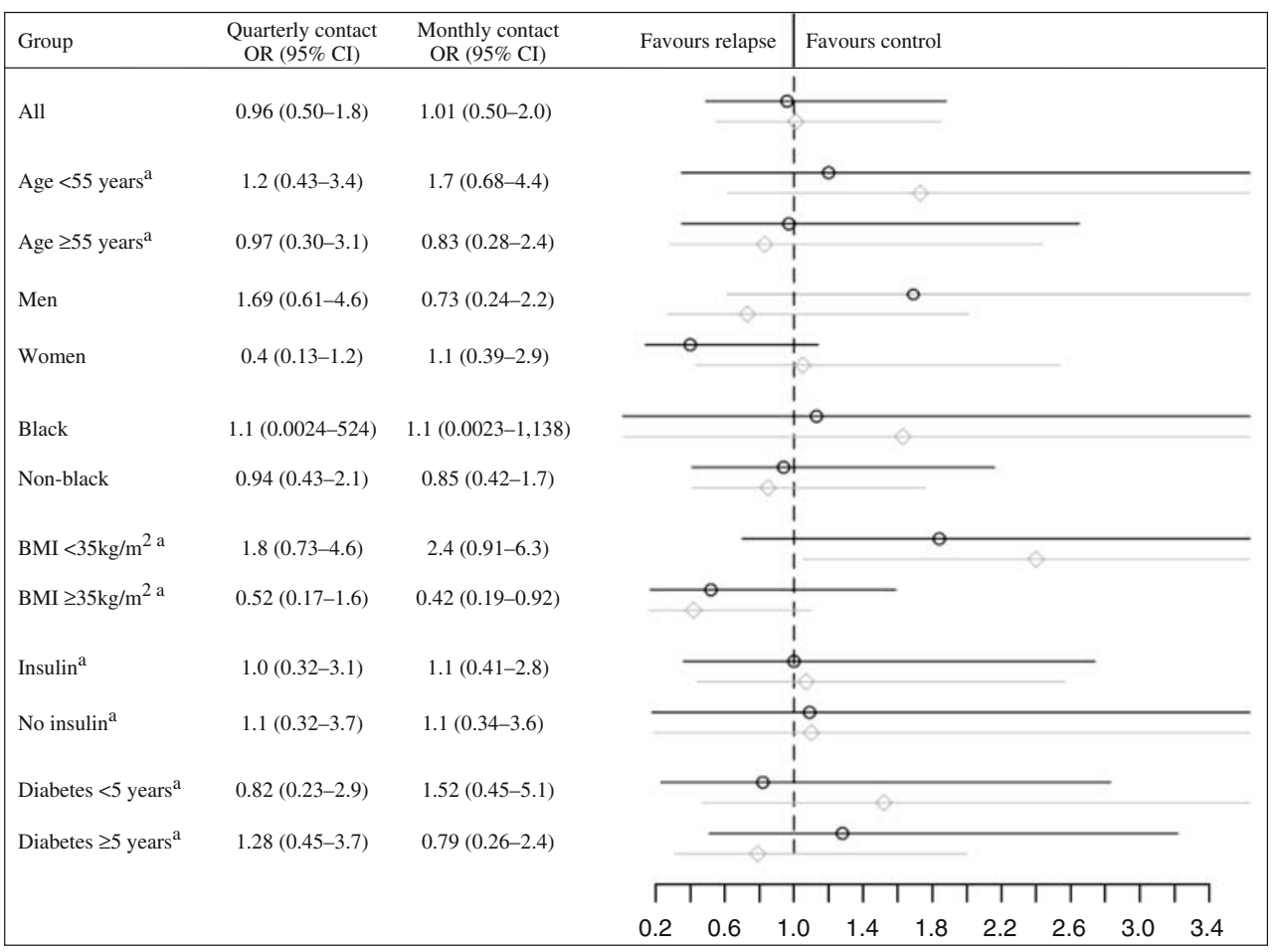


have impaired the patient's ability to act upon results of the telephone intervention during our maintenance intervention.

On the basis of complementary literature, frequent telephone contact was expected to reduce the probability of relapse in a dose dependent fashion $[9,16]$. Evidence from the literature on obesity suggests that regular telephone contact emphasising self-regulation of behaviour and providing feedback on self-monitoring records can be effective in delaying the onset of weight regain $[9,16]$. As a rule, however, it has not always improved long-term outcomes in comparison with usual care [17, 18]. Increasingly, disease management companies rely on the telephone to support patient self-management efforts [19]. The efficacy of these programmes is uncertain. The present study was designed with the disease management healthcare delivery model in mind. Patients had achieved glycaemic control at baseline and telephone support was provided by healthcare professionals who were able to adjust medications and were trained in cognitive behavioural techniques [5]. The intervention was delivered by experienced providers and was integrated in the patients' existing medical system. Despite this, the intervention did not prove sufficient for preventing glycaemic relapse.

In response to the need to improve chronic disease outcomes, the disease management industry has emerged as an intermediary between healthcare providers and patients. The general approach of these companies is to develop patient database systems and provide case management and patient education services to targeted patient populations [20]. As of 2006, more than $83 \%$ of the 500 largest employers in the USA had developed their own or contracted an external disease management company [4]. In 2003 to 2004, Medicare additionally provided fee-forservice contracts to eight disease management firms under a 3 year pilot programme [21]. Quasi-experimental and prepost analyses of health maintenance organisation-based programmes suggest that diabetes-focused disease management programmes have reduced costs and improved health outcomes [22, 23]. However, early indications from randomised controlled trials commissioned by the Centers for Medicare and Medicaid Services suggest that disease management programmes are not producing the expected improvements in health outcomes or reductions in costs [21]. Another approach is to improve medication adherence, which has also had mixed results [24]. The relative use of disease management programmes for quality improvement in diabetes care remains the subject of debate.

Our study has limitations. First, the appropriateness of the intervention is a possible concern. The intervention was modelled with attention to best practice in diabetes management and to relapse prevention programmes adopted for the treatment of obesity, alcohol, drug and smoking cessation. Other interventions may prove more effective. Second, the study protocol did not address: (1) how often the participants saw their primary care providers; (2) the care provided by the primary care providers; or (3) the counselling given in that setting. Such variables are often not controlled in disease management programmes, but do introduce the possibility of residual confounding. Third, the study protocol did not address care for participants who relapsed. By not having a specific protocol to address relapse, we may have missed an opportunity to reduce the prevalent proportions of relapse, although incident relapse would not have been affected. Fourth, our study was not powered to assess subgroup analyses and these results must be interpreted with caution. Fifth, the rate of relapse was much lower, nearly $50 \%$ less, than anticipated from the observational studies $[7,8]$. This may be due to the selection of a highly motivated population, who not only completed a 12 -week intensive improvement programme, but also successfully lowered their $\mathrm{HbA}_{1 \mathrm{c}} \geq 1 \%$. Finally, there may have been a powerful Hawthorne effect in the study. In this study we administered questionnaires that asked about the importance of self-management behaviours and participant self-efficacy in performing these behaviours. These questions are likely to have provided a powerful cue to action for all participants. The phenomenon of lower than anticipated event rates in the control arm has been seen in other behavioural intervention studies $[9,25]$.

Glycaemic relapse is an emerging area of focus in the diabetes literature. In this single-centre randomised controlled trial of newly controlled patients with type 2 diabetes, regularly scheduled telephone contact by a nurse practitioner was no more effective than routine follow-up care in preventing glycaemic relapse. The failure of this carefully crafted and integrated telephone intervention indicates that the relapse phenomenon needs critical reflection and that the current methods of disease management are likely to be insufficient to prevent glycaemic relapse. Future research should focus on identifying alternative delivery routes, dose adjustments and tailoring of relapse prevention interventions to patient-specific characteristics.

Acknowledgements The research was supported by the National Institute of Diabetes and Digestive and Kidney Disease R18 DK 062258, P60 DK 020593 and K24 DK 077875. M. M. Huizinga was supported by National Institute of Environmental Health Sciences 1 K12 ES 015855 and National Center for Research Resources 5 K12 RR 023266.

Duality of interest The authors declare that there is no duality of interest associated with this manuscript.

\section{References}

1. No authors listed (1998) Intensive blood-glucose control with sulphonylureas or insulin compared with conventional treatment and risk of complications in patients with type 2 diabetes (UKPDS 33). UK Prospective Diabetes Study (UKPDS) Group. Lancet 352:837-853 
2. Ford E, Li C, Little R, Mokdad A (2008) Trend in A1C concentrations among U.S. adults with diagnosed diabetes from 1999 to 2004. Diabetes Care 31:102-104

3. Institute of Medicine (2001) Crossing the quality chasm: a new health system for the 21 st century. National Academy Press, Washington

4. Mattke S, Seid M, Ma S (2007) Evidence for the effect of disease management: is \$1 billion a year a good investment? Am J Manag Care 13:670-676

5. Renders CM, Valk GD, Griffin S, Wagner EH, Eijk JT, Assendelft WJ (2001) Interventions to improve the management of diabetes mellitus in primary care, outpatient and community settings. Cochrane Database Syst Rev, Issue 4, Art. no: CD001481. doi:10.1002/14651858.CD001481

6. Akimoto M, Fukunishi I, Kanno K et al (2004) Psychosocial predictors of relapse among diabetes patients: a 2-year follow-up after inpatient diabetes education. Psychosomatics 45:343-349

7. Elasy TA, Graber AL, Wolff K, Brown A, Shintani A (2003) Glycemic relapse after an intensive outpatient intervention for type 2 diabetes. Diabetes Care 26:1645-1646

8. Graber AL, Shintani AK, Wolff K, Brown A, Elasy TA (2006) Glycemic relapse in type 2 diabetes. Endocr Pract 12:145-151

9. Svetkey LP, Stevens VJ, Brantley PJ et al (2008) Comparison of strategies for sustaining weight loss: the weight loss maintenance randomized controlled trial. Jama 299:1139-1148

10. Huizinga MM, Shintani A, Michon S et al (2006) A randomized controlled trial to prevent glycemic relapse in longitudinal diabetes care: study protocol (NCT00362193). Implement Sci 1:24

11. Quinn DC, Graber AL, Elasy TA, Thomas J, Wolff K, Brown A (2001) Overcoming turf battles: developing a pragmatic, collaborative model to improve glycemic control in patients with diabetes. Joint Comm J Qual Improv 27:255-264

12. Brown AW, Wolff KL, Elasy TA, Graber AL (2001) The role of advanced practice nurses in a shared care diabetes practice model. The Diabetes Educator 27:492-496, 498-500, 502

13. Graber AL, Elasy TA, Quinn D, Wolff K, Brown A (2002) Improving glycemic control in adults with diabetes mellitus: shared responsibility in primary care practices. South Med J 95:684-690
14. Pichert JW, Stetson BA (1994) Evaluating the treatment integrity of a continuing education program. J Nurs Staff Dev 10:75-80

15. Shojania KG, Ranji SR, McDonald KM et al (2006) Effects of quality improvement strategies for type 2 diabetes on glycemic control: a meta-regression analysis. Jama 296:427-440

16. Perri MG, McAdoo WG, McAllister DA et al (1987) Effects of peer support and therapist contact on long-term weight loss. J Consult Clin Psychol 55:615-617

17. Perri MG, Shapiro RM, Ludwig WW, Twentyman CT, McAdoo WG (1984) Maintenance strategies for the treatment of obesity: an evaluation of relapse prevention training and posttreatment contact by mail and telephone. J Consult Clin Psychol 52:404-413

18. Wing R, Jeffery R, Hellerstedt W, Burton L (1996) Effect of frequent phone contacts and optional food provision on maintenance of weight loss. Ann Behav Med 18:172-176

19. Piette J (2005) Using telephone support to manage chronic disease. Available from www.chcf.org/topics/chronicdisease/index.cfm? itemID $=111784$, accessed 23 December 2009

20. Casalino LP (2005) Disease management and the organization of physician practice. Jama 293:485-488

21. (2008) Fact sheet: Completion of Phase I of Medicare Health Support Program. Centers for Medicare and Medicaid Services. Available from http://www.cms.hhs.gov/CCIP/02_Highlights.asp and http://www.cms.hhs.gov/CCIP/downloads/EOP_Fact_Sheet FINAL 012808.pdf, accessed 23 December 2009

22. Sidorov J, Gabbay R, Harris R et al (2000) Disease management for diabetes mellitus: impact on hemoglobin A1c. Am J Manag Care 6:1217-1226

23. Villagra VG, Ahmed T (2004) Effectiveness of a disease management program for patients with diabetes. Health Aff (MillWood) 23:255-266

24. Haynes RB, Ackloo E, Sahota N, McDonald HP, Yao X (2008) Interventions for enhancing medication adherence. Cochrane Database of Systematic Reviews Issue 2. Art. no.: CD000011. doi:10.1002/14651858.CD000011.pub3

25. Appel LJ, Champagne CM, Harsha DW et al (2003) Effects of comprehensive lifestyle modification on blood pressure control: main results of the PREMIER clinical trial. Jama 289:2083-2093 